\title{
Brain Delivery of Curcumin Through Low-Intensity Ultrasound-Induced Blood-Brain Barrier Opening via Lipid-PLGA Nanobubbles
}

\author{
Yiran Yan, ', ${ }^{\prime}$ Yan Chen, ${ }^{2, *}$ \\ Zhongxun Liu,' Feiyan Cai, ${ }^{3}$ \\ Wanting Niu, 4,5 Liming Song,' \\ Haifeng Liang,' Zhiwen Su, 'Bo Yu, ' \\ Fei Yan $^{6}$ \\ 'Department of Orthopedics, Zhujiang \\ Hospital, Southern Medical University, \\ Guangzhou, Guangdong, 51 0282, People's \\ Republic of China; ${ }^{2}$ Department of Ultrasonic \\ Diagnosis, Zhujiang Hospital, Southern Medical \\ University, Guangzhou, Guangdong, 510282, \\ People's Republic of China; ${ }^{3}$ Paul C. Lauterbur \\ Research Center for Biomedical Imaging, \\ Institute of Biomedical and Health Engineering, \\ Shenzhen Institutes of Advanced Technology, \\ Chinese Academy of Sciences, Shenzhen, \\ Guangdong, 518055, People's Republic of \\ China; ${ }^{4} V A$ Boston Healthcare System, Boston, \\ MA, 02130, USA; ${ }^{5}$ Department of \\ Orthopedics, Brigham and Women's Hospital, \\ Harvard Medical School, Boston, MA, 02II5, \\ USA; ${ }^{6}$ CAS Key Laboratory of Quantitative \\ Engineering Biology, Shenzhen Institute of \\ Synthetic Biology, Shenzhen Institutes of \\ Advanced Technology, Chinese Academy of \\ Sciences, Shenzhen, Guangdong, 518055, \\ People's Republic of China \\ *These authors contributed equally to this \\ work
}

Correspondence: Fei Yan

Shenzhen Institutes of Advance Technology,

Chinese Academy of Science, 1068 Xueyuan

Avenue, Shenzhen University Town,

Shenzhen, Guangdong, 518055, People's

Republic of China

Tel +8675586392284

Fax +8675596382299

Email fei.yan@siat.ac.cn

Bo Yu

Department of Orthopedics, Zhujiang

Hospital, Southern Medical University, 253

Gongye Avenue, Haizhu District,

Guangzhou, Guangdong, 510280, People's

Republic of China

Tel +862067282573

Fax +862061643010

Email gzyubo@163.com
Background: Parkinson's disease (PD) is a progressive neurodegenerative disorder. Owing to the presence of blood-brain barrier (BBB), conventional pharmaceutical agents are difficult to the diseased nuclei and exert their action to inhibit or delay the progress of PD. Recent literatures have demonstrated that curcumin shows the great potential to treat PD. However, its applications are still difficult in vivo due to its poor druggability and low bioavailability through the BBB.

Methods: Melt-crystallization methods were used to improve the solubility of curcumin, and curcumin-loaded lipid-PLGA nanobubbles (Cur-NBs) were fabricated through encapsulating the curcumin into the cavity of lipid-PLGA nanobubbles. The bubble size, zeta potentials, ultrasound imaging capability and drug encapsulation efficiency of the Cur-NBs were characterized by a series of analytical methods. Low-intensity focused ultrasound (LIFU) combined with Cur-NB was used to open the BBB to facilitate curcumin delivery into the deep brain of PD mice, followed by behavioral evaluation for the treatment efficacy. Results: The solubility of curcumin was improved by melt-crystallization methods, with 2627-fold higher than pure curcumin. The resulting Cur-NBs have a nanoscale size about $400 \mathrm{~nm}$ and show excellent contrast imaging performance. Curcumin drugs encapsulated into Cur-NBs could be effectively released when Cur-NBs were irradiated by LIFU at the optimized acoustic pressure, achieving $30 \%$ cumulative release rate within 6 h. Importantly, Cur-NBs combined with LIFU can open the BBB and locally deliver the curcumin into the deep-seated brain nuclei, significantly enhancing efficacy of curcumin in the Parkinson C57BL/6J mice model in comparison with only Cur-NBs and LIFU groups.

Conclusion: In this work, we greatly improved the solubility of curcumin and developed Cur-NBs for brain delivery of curcumin against PD through combining with LIFU-mediating BBB. Cur-NBs provide a platform for these potential drugs which are difficult to cross the BBB to treat PD disease or other central nervous system (CNS) diseases.

Keywords: nanobubbles, blood-brain barrier, low-intensity focus ultrasound, curcumin, Parkinson's disease

\section{Introduction}

Parkinson's disease (PD) is a chronic and progressive neurodegenerative disorder characterized pathologically by death of midbrain dopaminergic neurons of substantia nigra pars compacta (SNC) and the formation of intraneuronal inclusions called Lewy bodies, which are mainly comprised of alpha-synuclein (a-syn). ${ }^{1-3}$ Although supplying endogenous source of dopamine is the current pharmacological therapy to treat $\mathrm{PD}$, it is not neuroprotective and the continued neuronal 
degeneration ultimately leads to recurrence of symptoms. ${ }^{4}$ Nowadays, multiple pathogenic mechanisms have been demonstrated to be involved in $\mathrm{PD},{ }^{5,6}$ and many emerging novel therapies were developed to target to those pathogenic mechanisms. ${ }^{7-9}$ However, many potential PD pharmacological agents have no or limited therapeutic efficacies due to their low druggability and poor permeation across blood-brain barrier (BBB). ${ }^{10-12}$ Therefore, there is an urgent demand to find novel strategies for improving their druggability and favoring their brain delivery in vivo with minimal adverse effects in the central nervous.

In recent years, the combination of microbubbles (MBs) with transcranial low-intensity focus ultrasound (LIFU) is developed as a promising strategy to overcome BBB and locally deliver drugs against central nervous diseases. ${ }^{13,14}$ In this technology, the beam excited by low-frequency transducer can transmit into the skull and be focused on the special brain region or nuclei, inducing the MBs circulating in these brain regions to generate cavitation, such as stable cavitation and inertial cavitation. ${ }^{15}$ The former includes microstreaming, radiation force and the latter is involved with micro jets, shear forces and shockwave. ${ }^{16}$ Both stable cavitation and inertial cavitation collectively exert strong mechanical effects on the cerebral blood vessels, leading to the transient and revisable BBB opening. ${ }^{17}$ Compared to other brain drug delivery strategies such as endothelial cell permeability by mannitol, ${ }^{18}$ receptor-mediated endocytosis/transcytosis ${ }^{19}$ and nose-to-brain delivery, ${ }^{20}$ LIFU-induced blood-brain barrier opening possesses many obvious advantages, including noninvasiveness, local and efficient drug delivery and suitable for both small and large molecular drugs. ${ }^{21}$ Moreover, some drugs can be coated onto the surface of MBs via electrostatic interaction, or encapsulated into the bubble shell through hydrophobic interaction. ${ }^{22}$ By this way, drugs can be prevented from exogenous enzyme degradation and expand their druggability, greatly increasing their availability. Traditionally, MBs have a large particle size range from 1 $\mu \mathrm{m}$ to $8 \mu \mathrm{m}$ in diameter, failing to shuttle freely in liver and spleen. ${ }^{23}$ Also, lipid-based MBs have a poor drug encapsulation efficiency due to their monolayer lipid shell structure. ${ }^{24}$ Recently, nanobubbles with nanoscale size $(<1000 \mathrm{~nm})$ are attracting researchers' attention due to some unique advantages such as higher drug encapsulation efficiency, more stable and longer circulation in blood. Thus, it is desirable to develop a kind of nano-size bubbles with high drug encapsulation efficiency for LIFU-mediated BBB opening to treat neurodegenerative disorder.
Curcumin, a polyphenols compound separated from the rhizoma of Zingiberaceae, has diverse biological effects on anti-tumor, bacteriostasis, antioxidant, and antiinflammatory. ${ }^{25}$ Curcumin has been shown to its great potential in preventing many different types of cancers, including multiple myeloma and colorectal, pancreatic, breast, prostate, lung, head, and neck cancers. ${ }^{26}$ In addition, curcumin is also reported as neuroprotective agents in treating neurological disorders. ${ }^{27-29}$ Literatures in which performed different experimental PD models in vitro also revealed that curcumin could inhibit and remove a-syn effectively, leading to attenuation of a-syn oligomer toxicity in cells. ${ }^{30,31}$ In addition, it has confirmed that curcumin was non-toxic even at high doses. However, curcumin possesses low bioavailability due to its poor solubility in aqueous solution $(20.0 \mu \mathrm{g} / \mathrm{mL}$ in water $),{ }^{32}$ and poor permeability to the BBB. ${ }^{33}$ Solid dispersion is a state of fine grain dispersion in highly soluble carriers for improving the solubilization of insoluble drugs via various approaches, including spray drying, solvent evaporation and hot melt extrusion. ${ }^{34}$ PEG-6K is a noxious and highly soluble agent that can be widely used for solid dispersion through melt-crystallization method. To address the above problems, we used melt-crystallization methods to improve the solubility of curcumin and fabricated curcumin-loaded Lipid-PLGA hybrid nanobubbles (Cur-NBs) in this study. Owing to the relative hard shell and uniform size distribution of Cur-NBs in comparison with lipid bubbles, stable cavitation for BBB opening and drug release for brain delivery would be controlled better by regulating the input of acoustic energy. The Cur-NBs were used to induce BBB opening by combining with LIFU and the efficacy against PD was evaluated. Our study provides a novel strategy to noninvasively, safely, locally deliver the curcumin into the mouse brain to treat $\mathrm{PD}$ via ultrasound-induce BBB opening (Figure 1).

\section{Materials and Methods Materials}

50:50 poly(lactide-co-glycolide) (PLGA), Methylene chloride, and ammonium bicarbonate were obtained from Jinan Daigang Biomaterial Co., Ltd (Jinan, Shandong, China). 1,2-distearoyl-sn-glycero-3-phosphatidylcholine (DSPC) and 1,2-distearoyl-sn-glycero-3-phosphoethanola-mine$\mathrm{N}-[$ methoxy (polyethylene glycol)-2000] (DSPE-PEG2000) were acquired from Avanti Polar Lipids Inc. (Alabaster, AL, USA). Polyvinyl alcohol (PVA, Mw, 30,000-70,000), 

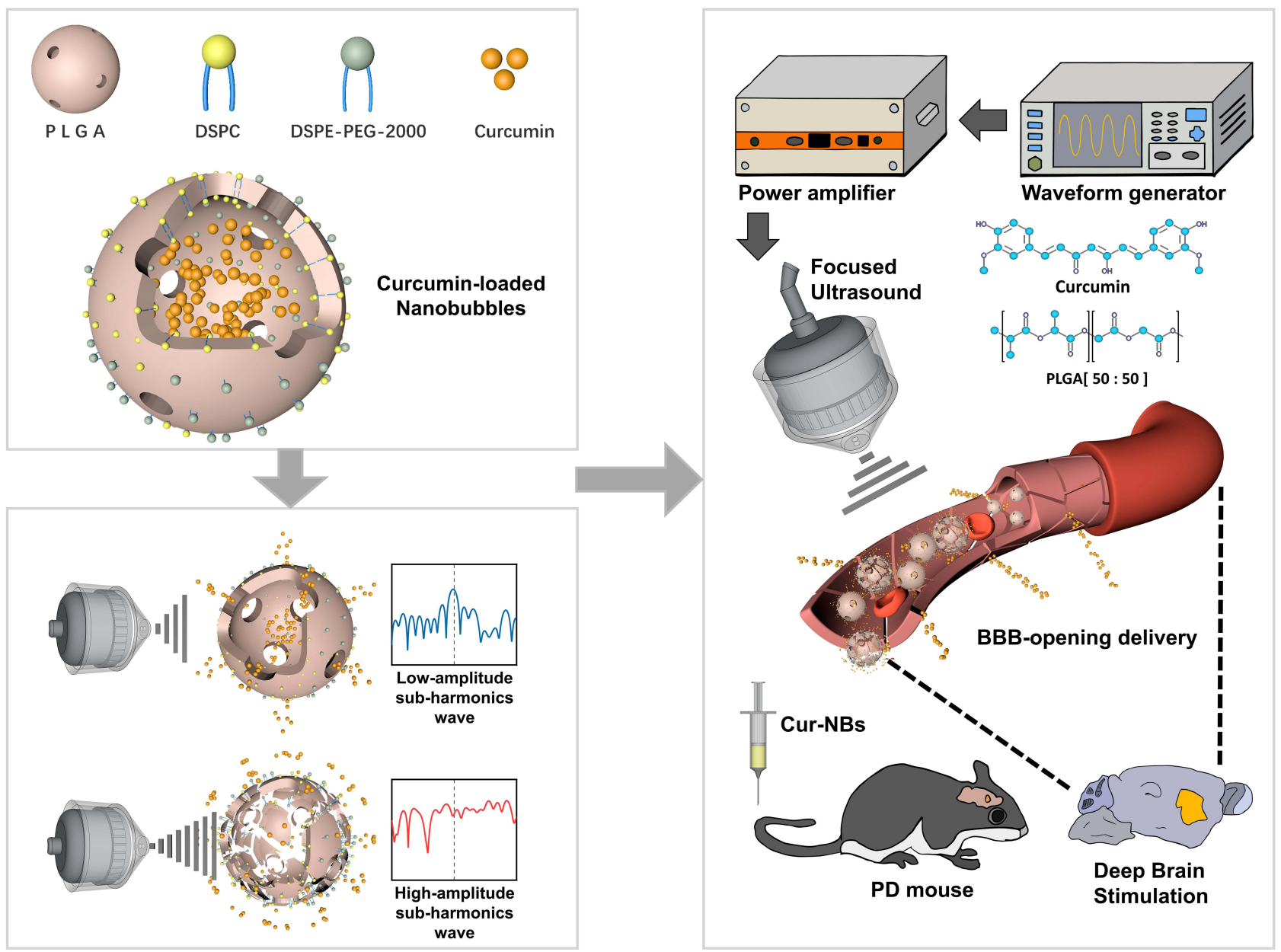

Figure I Schematic illustration of the noninvasive localized delivery of curcumin by Cur-NBs combined with LIFU for PD therapy.

ammonium bicarbonate $\left(\left(\mathrm{NH}_{4}\right) \mathrm{HCO}_{3}\right)$, pentobarbital sodium, Curcumin (Cur), Evans blue (EB) and 1-Methyl4-phenyl-1,2,3,6-tetrahydropyridine (MPTP) were purchased from Sigma Aldrich (St Louis, MO, USA). Polyethylene glycol 6000 (PEG-6000) was purchased from Solarbio Co., Ltd. (Beijing, China). All other chemicals were of analytical grade and used without any further purification. Female C57BL6 mice, aged 6-8 weeks (weighing approximately $20 \mathrm{~g}$ ), were purchased from Guangdong Medical Experimental Animal Center (Guangzhou, Guangdong, China).

\section{Preparation of PEG Modified Curcumin Solid Dispersion (SD) and Physical Mixture (PM)}

Solid dispersions were prepared with Curcumin and PEG6000 at weight ratios of $1: 10$ for future research by meltcrystallization methods. $^{35,36}$ Briefly, the mixture of Curcumin and PEG-6000 were heated in a water bath at $65^{\circ} \mathrm{C}$ to achieve complete melting initially, while stirring for $0.5 \mathrm{~h}$ to obtain a clear homogeneous solution. The resulting homogeneous liquid was shock-frozen in the centrifugation tube which was immersed in liquid nitrogen for $5 \mathrm{~min}$ to form the PEG-modified curcumin solid dispersion (SD-Cur), and then the SD-Cur were collected and ground to a powder. The curcumin-PEG6k physical mixture (PM-Cur) was obtained by mixing the same amount of curcumin and PEG-6000 in a mortar for $10 \mathrm{~min}$. The samples were stored at $25^{\circ} \mathrm{C}$ before use.

\section{Curcumin Release from SD-Cur}

To evaluate the release behavior of various mixture mode, $5 \mathrm{~mL}$ samples of pure curcumin, PM-Cur or SD-Cur with $5 \mathrm{mg}$ curcumin in PBS were added to dialysis membranes (MW 100000 Da, Yuanye, Shanghai, China) against PBS and rotated at the speed of $50 \mathrm{rpm}$ at $37^{\circ} \mathrm{C} .1 \mathrm{~mL}$ of each sample solution was collected at varied time points $(5,15$, $30,60,90$ and $120 \mathrm{~min}$ ), to analyze the real-time 
concentration by microplate reader (Infinite M1000 PRO, Tecan, Männedorf, Switzerland) and $1 \mathrm{~mL}$ of PBS was then replenished. The released drugs were isolated by centrifugation at $4500 \mathrm{~g}$ for $10 \mathrm{~min}$ and the $100 \mu \mathrm{L}$ clear liquid was transferred to a 96-well plate for determining the drug concentrations by microplate reader. The cumulative release rate was calculated according to the formula:

$$
\begin{gathered}
\text { Cumulative amount released (\%) } \\
=\frac{C_{n} V+\sum_{i=1}^{n-1} C_{i} V_{i}}{W_{C u r}} \times 100 \%
\end{gathered}
$$

\section{Preparation of Cur-NBs}

The Cur-NBs were prepared by using a slight modification of the double emulsion evaporation process. Briefly, the SD-Cur were synthesized as previously described using melt-crystallization methods firstly at weight ratio of 1:10. Then, $55 \mathrm{mg}$ SD-Cur was dissolved in $1 \mathrm{~mL}$ doubly distilled water. After that, $60 \mathrm{mg}$ ammonium bicarbonate was added to this SD-Cur solution as aqueous phase. Fiftymilligram PLGA (50:50), $2 \mathrm{mg}$ DSPC and $0.5 \mathrm{mg}$ DSPEPEG2000 were dissolved in $1 \mathrm{~mL}$ dichloromethane (DCM, Lingfeng Chemical Reagent Co., Ltd, Shanghai, China) at room temperature. $0.2 \mathrm{~mL}$ freshly prepared aqueous phase was added to the former oil phase. The mixed solution was emulsified by sonication (130 W, Sonics and Materials, Newtown, CT, USA) for $2 \mathrm{~min}$ ( $40 \%$ amplitude, $30 \mathrm{~ms}$ on, $30 \mathrm{~ms}$ off) with an ice-water bath, and then $5 \mathrm{~mL} 3.5 \% \mathrm{w} /$ $\mathrm{v}$ of PVA aqueous solution was added to the initial emulsion and homogenized by homogenizer (T25 digital, IKA, Staufen, Germany) with $7000 \mathrm{rpm}$ for $6 \mathrm{~min}$. The generated emulsion was homogenized at $7000 \mathrm{rpm}$ for 6 min immediately following $8 \mathrm{~mL}$ doubly distilled water addition. The final suspension was then stirred at room temperature for $4 \mathrm{~h}$. Cur-NBs were separated subsequently by centrifugation at $4000 \mathrm{~g}$ for $10 \mathrm{~min}$. The obtained nanobubbles were resuspended in doubly distilled water, rinsed for 3 times, and lyophilized for $48 \mathrm{~h}$. Finally, the samples were refilled with air and stored at $4{ }^{\circ} \mathrm{C}$.

\section{Characterization of Cur-NBs}

The morphology of Cur-NBs was confirmed using a scanning electron microscope (SEM) (HITACHI SU8010, Hitachi, Tokyo, Japan). The inner structure of Cur-NBs was characterized by a transmission electron microscope (TEM) (FEI spirit T12, FEI Company, Hillsboro, OR, USA). Briefly, the prepared Cur-NBs were diluted at distilled water to a make concentrations suitable concentration for the convenience of observing, following followed by dropping into onto the TEM copper grid. Then, $1 \%$ phosphotungstic acid solution was dropped added onto the samples for $2 \mathrm{~min}$ and dropwise then washed with PBS on filter paper. The solvent of sample was evaporated off and the residual solid nanobubbles was dried overnight before TEM observations. The average diameter and size distribution of the Cur-NBs was characterized using dynamic light scattering (DLS) (MS2000, Malvern Instruments Ltd., Malvern, Worcestershire, UK). To determine the fluorescence excitation spectra changes between the curcumin itself and the rehydration solution of Cur-NBs, FS5 spectrofluorometer (Edinburgh Instruments, Edinburgh, UK) was used, and fluorescence imaging was visualized using a confocal laser scanning microscope (A1R, Nikon, Tokyo, Japan). To observe the floating behavior of nanobubbles, resuspended solution of Cur-NBs was left to stand at room temperature for $5 \mathrm{~h}$, and a camera was used to record the floatation.

\section{Hemolysis Assay of Cur-NBs}

Serum was removed from mouse blood by centrifugation to obtain working solution of mouse red blood cells (RBC). After three washes with PBS, the cells were diluted 10-folds with PBS solution for further testing. Then, (a) the negative control which was mixed with $0.2 \mathrm{~mL}$ diluted $\mathrm{RBC}$ suspension in $0.8 \mathrm{~mL}$ PBS was prepared, (b) the positive control which was mixed with $0.2 \mathrm{~mL}$ diluted $\mathrm{RBC}$ suspension in $0.8 \mathrm{~mL}$ deionized water was also prepared, and (c) $0.8 \mathrm{~mL}$ Cur-NBs solution with various solution concentration of 25, 50, 100, 200, 400, $800,1600 \mu \mathrm{g} / \mathrm{mL}$ were mixed with $0.2 \mathrm{~mL}$ diluted $\mathrm{RBC}$ suspension. The supernatant was acquired by centrifugation followed the incubation of above mixture for 3 hours. Based on the absorbance values detected by an UV-Vis spectrophotometer (UV-2600, Shimadzu Corporation, Tokyo, Japan) at the wavelength of $450 \mathrm{~nm}$, the percentage of hemolysis was calculated according to the following formula:

$$
\text { Hemolysis }(\%)=\frac{A b_{\text {sample }}-A b_{\text {negative }}}{A b_{\text {positive }}-A b_{\text {negative }}} \times 100 \%
$$

\section{In vitro Drug Release from Cur-NBs}

To evaluate the drug release behavior of nanobubbles by LIFU, $1 \mathrm{mg} / \mathrm{mL}$ Cur-NBs were stimulated by various acoustic intensity of $0,0.31,0.38$ and $0.45 \mathrm{MPa}$ for 60 sec at $37{ }^{\circ} \mathrm{C}$. The LIFU system was composed with 
a spherical transducer (1.0 MHz; focal length: $38.0 \mathrm{~mm}$ ), a waveform generator (DG4000, Rigol, Beijing, China), and a $50 \mathrm{~dB}$ power amplifier (LZY-22+, Mini-circuits, New York, USA). We separated the released drugs from Cur-NBs by centrifugation at $4500 \mathrm{~g}$ for $10 \mathrm{~min}$ and the $100 \mu \mathrm{L}$ clear liquid was transferred to a 96-well plate for determining the drug concentrations by microplate reader. The real-time B-mode and contrast mode images were acquired using Vevo2100 Imaging System (Visual Sonics, Toronto, Ontario, Canada), following by observation under a scanning electron microscope to observe the destruction of Cur-NBs.

\section{Acoustic Power Spectrum of Cur-NBs}

To find out the optimal acoustic pressure to induce the stable cavitation and avoid inertial cavitation, we investigated the subharmonic power spectrum produced by cavitation of nanobubbles using the device setup as shown in Figure 4D. The passive cavitation detection (PCD) ${ }^{37}$ special monitoring platform for ultrasonic cavitation open $\mathrm{BBB}$ is composed of waveform generator, power amplifier, preamplifier (model 600, Millar Instruments, Houston, TX, USA), ultrasound transducer (V310-SU, Olympus, New York, USA) and digital oscilloscope (DPO4034, Tektronix, Beaverton, OR, USA). The ultrasonic transmitting transducer was fixed and connected with the waveform generator and the power amplifier, and the ultrasonic receiving transducer is connected with the preamplifier and the digital oscilloscope. Before each test, 100 ul Cur-NBs solution was added into the membrane chamber which contains $200 \mathrm{~mL}$ PBS. A $1 \mathrm{MHz}$ transducer was used to emit ultrasound wave to excite the Cur-NBs and another same transducer placed at a relative right-angle position was used to receive the acoustic spectrum signals.

\section{In vitro Ultrasound Imaging}

The ultrasound imaging capability of Cur-NBs was detected by placing varied concentrations of Cur-NBs (0, 0.0625, $0.125,0.25$ and $0.5 \mathrm{mg} / \mathrm{mL})$ into $1.5 \%(\mathrm{w} / \mathrm{v})$ agarose phantom holes. An $18 \mathrm{MHz}$ transducer which attached with Vevo 2100 imaging system was then positioned against the side of the holes to acquire the image containing B-mode and contrast mode. The actual intensity of the signals was calculated by instrument software of Vevo 2100.

\section{In vivo Ultrasound Imaging of Cur-NBs}

Before the experiment of in vivo ultrasound imaging experiment, a heating blanket was utilized to maintain the body temperature of mouse at $37.0^{\circ} \mathrm{C}$, and the fur on the head hair was removed and skin incision was performed to expose the skull after stabilization of anesthesia. Using an appropriate micro drill (RWD Life Science, Shenzhen, Guangdong, China) at a constant speed, a craniectomy was partially performed in the region ranged between bregma and lambda. The ultrasound probe was perpendicular to the plane of removed region and fixed to the fixator after subtle adjustments for better imaging. Owing to the hair, skin and skull had partially removed, superficial imaging mode was applied with a $7 \mathrm{MHz}$ ultrasound probe (Resona 7, Mindray, Shenzhen, Guangdong, China), while both the contrast-mode images and B-mode images were immediately collected by the Resona 7 ultrasound system at the following setting: depth at $2.5 \mathrm{~cm}$, Contrast-mode Gain at 65\%, B-mode gain at 55\%, frequency of contrast harmonic $(\mathrm{FCH})$ at $5.6 \mathrm{MHz}$, dynamic range at 115 $\mathrm{dB}$. The deep brain site of ultrasound irradiation was localized on the underneath the projected surface of the dorsal and lateral cortex, including striatum and substantia nigra with the help of stereotaxic apparatus (68030, RWD Life Science, Shenzhen, Guangdong, China). The setting of ultrasound probing parameter, including position, plane and depth remained unchanged during the whole imaging process.

\section{Brain-Blood Barrier Opening}

To evaluate the brain-blood barrier opening capacity of CurNBs combined with LIFU, mice's heads were depilated under $1 \%$ pentobarbital anesthesia, and were then fixed on a commercial stereotaxic apparatus to mark the localization of striatum in mouse brain. A focused ultrasound transducer (1.0 MHz; focal length: $38 \mathrm{~mm}$ ) which was connected with waveform generator and power amplifier was mounted on the external fixation device and was aligned with former target localization mark. The LIFU-pulse duration was about 200 $\mathrm{ms}$, and the pulse rate was $1 \mathrm{~Hz} .1 \mathrm{~min}$ ultrasound irradiation with varied acoustic pressure $(0.24-0.45 \mathrm{MPa})$ was performed on one side of deep brain by above fixed transducer following the mixed solution of Cur-NBs and Evans blue administration through tail vein. BBB opening was confirmed by observation with fluorescent microscopy through histological sections after $4 \mathrm{~h}$ of ultrasound irradiation.

\section{Treatment of PD Mouse}

The sub-acute mouse PD model was built through daily intravenous administration with MPTP for 7 days. Curcumin was delivered once every two days through LIFU-mediated BBB opening with Cur-NBs. The behavioral tests were performed at day 7 and day 21 . 
The C57BL/6 mice were divided randomly into 5 groups of 6 mice (healthy control, MPTP, LIFU/MPTP, Cur-NBs/ MPTP and LIFU/Cur-NBs/MPTP) and were subjected to a subacute PD modeling regimen which were built through daily intravenous administration with MPTP $(40 \mathrm{mg} / \mathrm{kg})$ for 7 days consecutively. Meanwhile, for the groups with Cur$\mathrm{NBs}$, the administration of Cur-NBs solution was performed once every other day for 6 times. For the groups with LIFU, ultrasound-induced BBB opening was performed once every other day for 6 times following Cur-NBs administration. The behavioral tests were performed at day 7 and day 21 after the first MPTP injection.

\section{Behavioral Tests}

The behavioral tests were used to assess the PD symptoms remission of the C57BL/6 mice with various treatments at day 7 and 21 . The elapsed time of the rotary rod test that each mouse walks on the constant rotarod (YLS-4C, Zhenghua Bio-instruments, Huaibei, Anhui, China) at a speed of $25 \mathrm{rpm}$ was recorded at least 3 times following training once to assess motor ability and coordination of mice among all groups. The run duration time of poleclimbing test that each mouse climb from the top of a $50 \mathrm{~cm}$ pole $(1 \mathrm{~cm}$ diameter) to the bottom was recorded at least 3 times to observe the neurogenic movement disorders of mice among all groups.

\section{Statistical Data Analysis}

The experimental data were present as the mean \pm standard error of mean (SEM) by using OriginLab 2018. Independent sample $t$-test and one-way ANOVA were calculated to analyze the significance of the difference between the groups by using SPSS Statistics software $\left({ }^{* *} P<0.01,{ }^{* * *} P<0.001 ;{ }^{\# \#} P<0.01,{ }^{\# \# \#} P<0.001\right.$; ${ }^{\Delta} P<0.05 ;$ ns $=$ not significant $P>0.05$ ).

\section{Results}

\section{Preparation of PEG-Modified Curcumin Solid Dispersion and Cur-NBs}

Curcumin has low solubility in physiological solution, which makes it difficult to be used for most pharmaceutical formulations. ${ }^{38}$ In order to improve the solubility of Curcumin, we first utilized manual grinding and meltcrystallization methods to obtain the PEG-modified curcumin solid dispersion (SD-Cur) (Figure 2A). An orange solid appearance could be observed after melt-crystallization (Figure 2B). Significantly improved solubility of
A

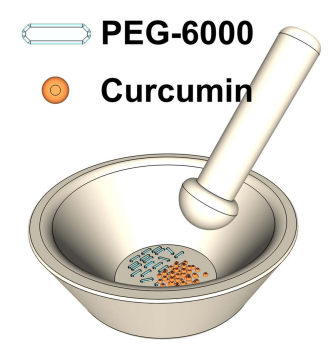

D

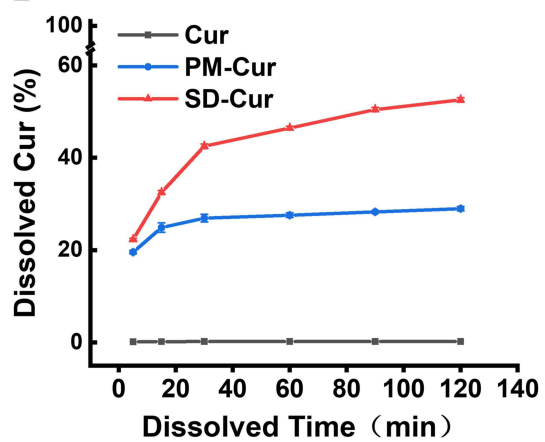

B

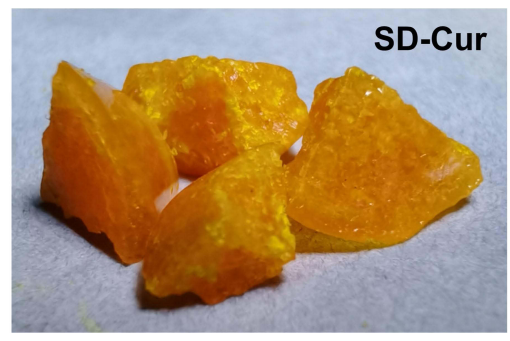

C

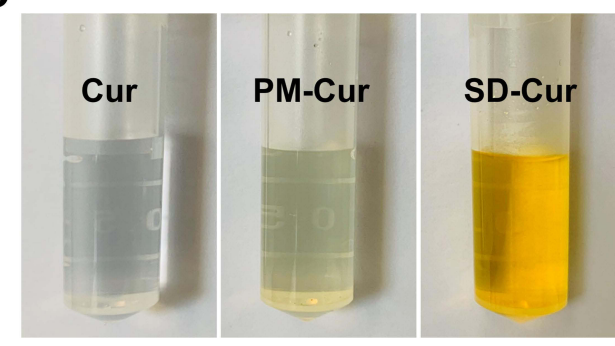

E

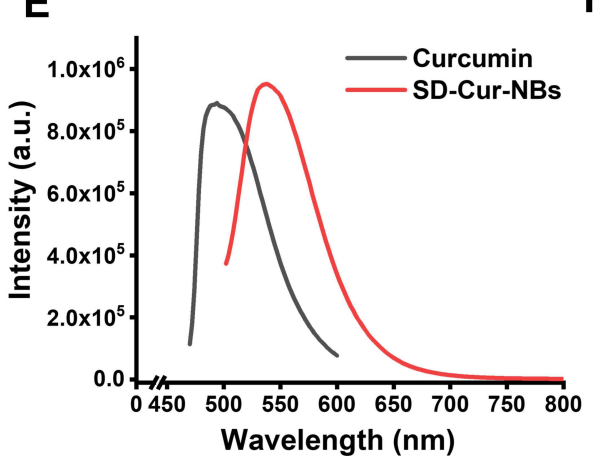

$\mathbf{F}^{\prime}$

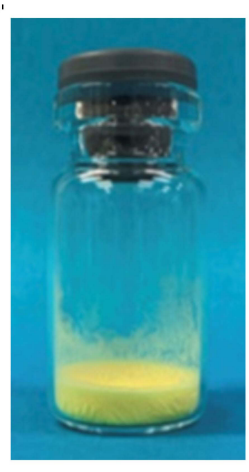

G

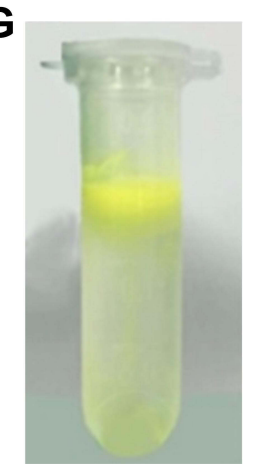

Figure 2 Preparation of PEG-modified curcumin solid dispersion and Cur-NBs. (A) Schematic illustration of the preparation of Cur-PEG solid dispersion using melt maxing method. (B) The resulting PEG-modified curcumin solid dispersion (SD-Cur). (C) The curcumin solution samples with pure curcumin, curcumin-PEG6k physical mixture (PM-Cur) and PEG-modified curcumin solid dispersion (SD-Cur). (D) The percent ratios of dissolved curcumin over time from different formulations. (E) The shift of fluorescence emission wavelength between curcumin and SD-Cur. (F) The resulting Cur-NBs in lyophilized powder. (G) The resulting Cur-NBs resuspended in in PBS. 
curcumin in PBS was achieved after melt-crystallization treatment, showing a darker color compared to curcumin and curcumin-PEG6k physical mixture (PM-Cur) (Figure 2C). The solubility of curcumin in SD-Cur aqueous solution was $52.54 \%$ which was 1.82 -fold higher than the solubility of PM-Cur (28.9\%), and 2627-fold higher than pure curcumin made a great enhancement to the solubilization of pure curcumin (0.02\%) after 2 $\mathrm{h}$ (Figure 2D). The slight red shift in the SD-Cur products fluorescence emission band was observed (emission wavelength: $550 \mathrm{~nm}$ ) in comparison to the pure curcumin solution (emission wavelength: $495 \mathrm{~nm}$ ) (Figure 2E). After that, the dissolved curcumin was used to fabricate the curcumin-loaded Lipid-PLGA nanobubbles (Cur-NBs) by using a slight modification of the double emulsion evaporation process and the resulting Cur-NBs are presented in Figure 2F. Obviously, the yellow Cur-NBs can float in the PBS solution, showing the Cur-NBs were successfully obtained (Figure 2G).

\section{Characterization of Cur-NBs}

A series of characterization methods were adopted to investigate the physical and chemical properties of CurNBs. Spherical morphology with porous architecture was observed by SEM and the hollow structure of Cur-NBs was revealed by TEM (Figure 3A and B). The encapsulation efficiency of Cur-NBs is $88.23 \%$ while drug loading content of Cur-NBs is $1.76 \%$. Cur-NBs had a size of $436 \pm$ $58.3 \mathrm{~nm}$ (PDI: $0.31 \pm 0.16$ ) (Figure 3C) and a zeta potential of $-27.5 \pm 0.6 \mathrm{mV}$ by dynamic light scattering (DLS) analysis. Moreover, Cur-NBs could keep stable at least 3 weeks since their size and zeta potential almost remained unchangeable (Figure 3D). Additionally, the data of particle size and the zeta potential occurred a slight change with diverse ratios of PLGA to SD-Cur in synthesis process. As shown in fluorescence microscopy images, green fluorescence could be observed in the internal cavity of Cur-NBs, proving that curcumin was entrapped into the bubbles (Figure 3E). Biocompatibility was confirmed by the hemolysis experiment, showing Cur-NBs had an excellent biocompatibility, even when the concentration reached $1600 \mu \mathrm{g} / \mathrm{mL}$ (Figure 3F and G).

\section{Acoustic Characteristics of Cur-NBs}

In order to test the ultrasound-mediated bubble destruction capability, Cur-NBs were exposed to $0,0.31,0.38$ or 0.45 $\mathrm{MPa}$ acoustic negative pressure for $1 \mathrm{~min}$. Significantly reduced contrast signals were found in these ultrasound- irradiated Cur-NBs (Figure 4A, middle). SEM images revealed obvious bubble destruction in the Cur-NBs which received with $0.45 \mathrm{MPa}$ acoustic exposure compared with these received with $0.31 \mathrm{MPa}$ (Figure 4A, bottom). Quantitative analyses of acoustic signal intensities of Cur-NBs showed the higher acoustic negative pressure was used, the lower acoustic signals would be (Figure 4B). In order to test the ultrasound-mediated drug release capability, Cur-NBs were exposed to ultrasound irradiation at $0.38 \mathrm{MPa}$ acoustic negative pressure. Compared with Cur-NBs which did not receive ultrasound irradiation, significantly improved drug release efficiency was found in the irradiated Cur-NBs (Figure 4C).

The appearance of subharmonics and broadband noise was deemed to be able to differentiate the signature of cavitation events (stable cavitation or inertial cavitation). Given the fact that the inertial cavitation is associated with the emission of broadband noise, the stable cavitation is evidenced by the radiation of the fundamental, higher harmonic, and sometimes subharmonic frequencies. ${ }^{39}$ To further examine the acoustic spectrum of Cur-NBs excited by different ultrasound energies, a passive cavitation detection (PCD) system was designed and constructed as shown in Figure 4D. Here, a $1 \mathrm{MHz}$ transducer was used to emit ultrasound wave to excite the Cur-NBs and another same transducer placed at a relative right-angle position was used to receive the acoustic spectrum signals. Obviously, the peak amplitude of acoustic spectrum increased with the acoustic intensities for Cur-NBs when the acoustic intensity was increased from $0.31 \mathrm{MPa}$ to $0.45 \mathrm{MPa}$. Interestingly, a broadband noisy spectrum for Cur-NBs at subharmonic frequencies was more obvious at $0.45 \mathrm{MPa}$, showing that there were more bubbles occurring inertial cavitation (Figure 4E).

\section{Ultrasound Imaging of Cur-NBs}

To determine the ultrasound imaging capability of Cur-NBs, we conducted the in vivo and in vitro imaging experiments. Various concentrations from 0 to $0.5 \mathrm{mg} / \mathrm{mL}$ of Cur-NBs were added into the agar phantom wells and imaged by ultrasound. Results showed that both B-mode imaging signals and the ultrasound contrast signals were gradually increased along with the bubble concentrations (Figure $5 \mathrm{~A}-\mathrm{C}$ ). The in vivo imaging was also detected in the brains of mice whose skull were partly removed to facilitate the ultrasound beam to transmit into the brain. The contrast-enhanced ultrasound echo signals of those Cur-NBs could be observed immediately after being 


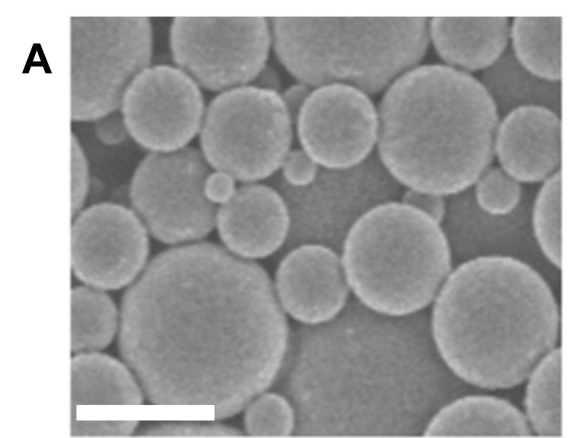

B

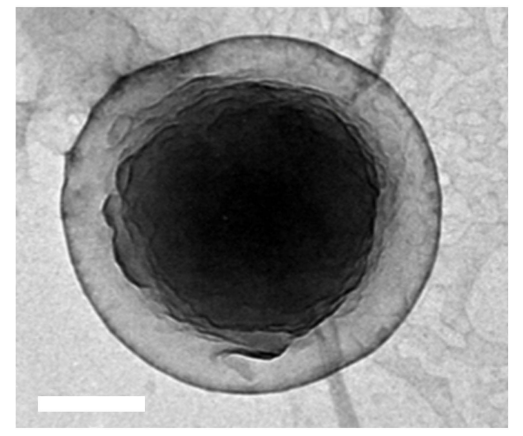

$\mathbf{E}$

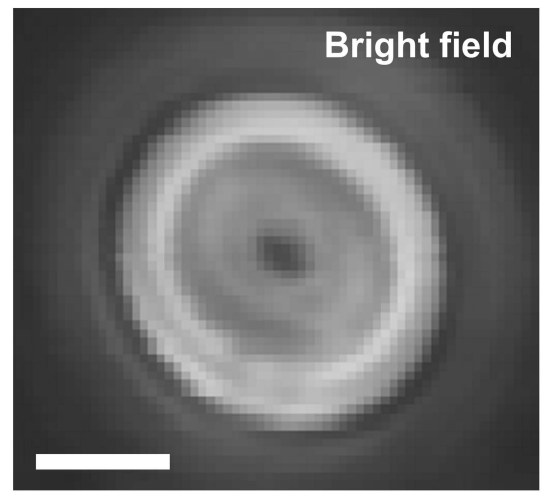

$\mathbf{F}$

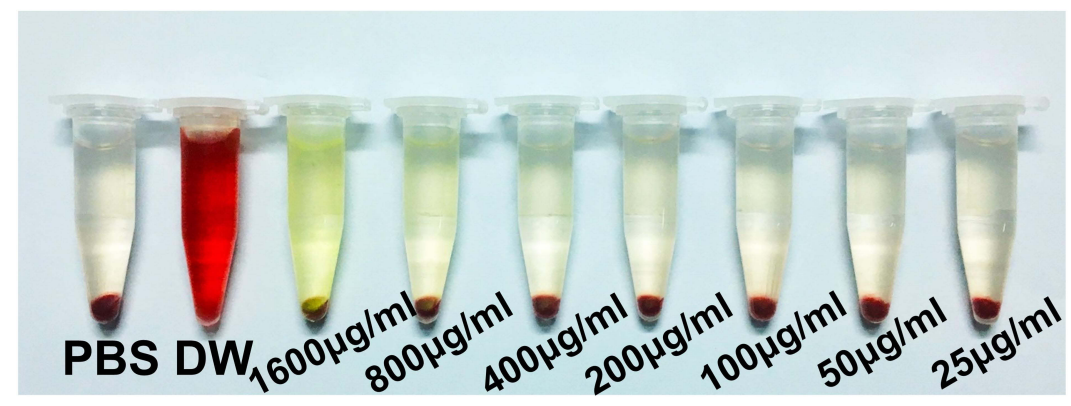

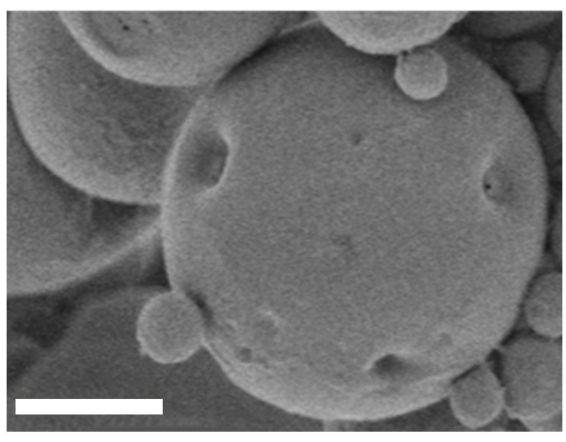
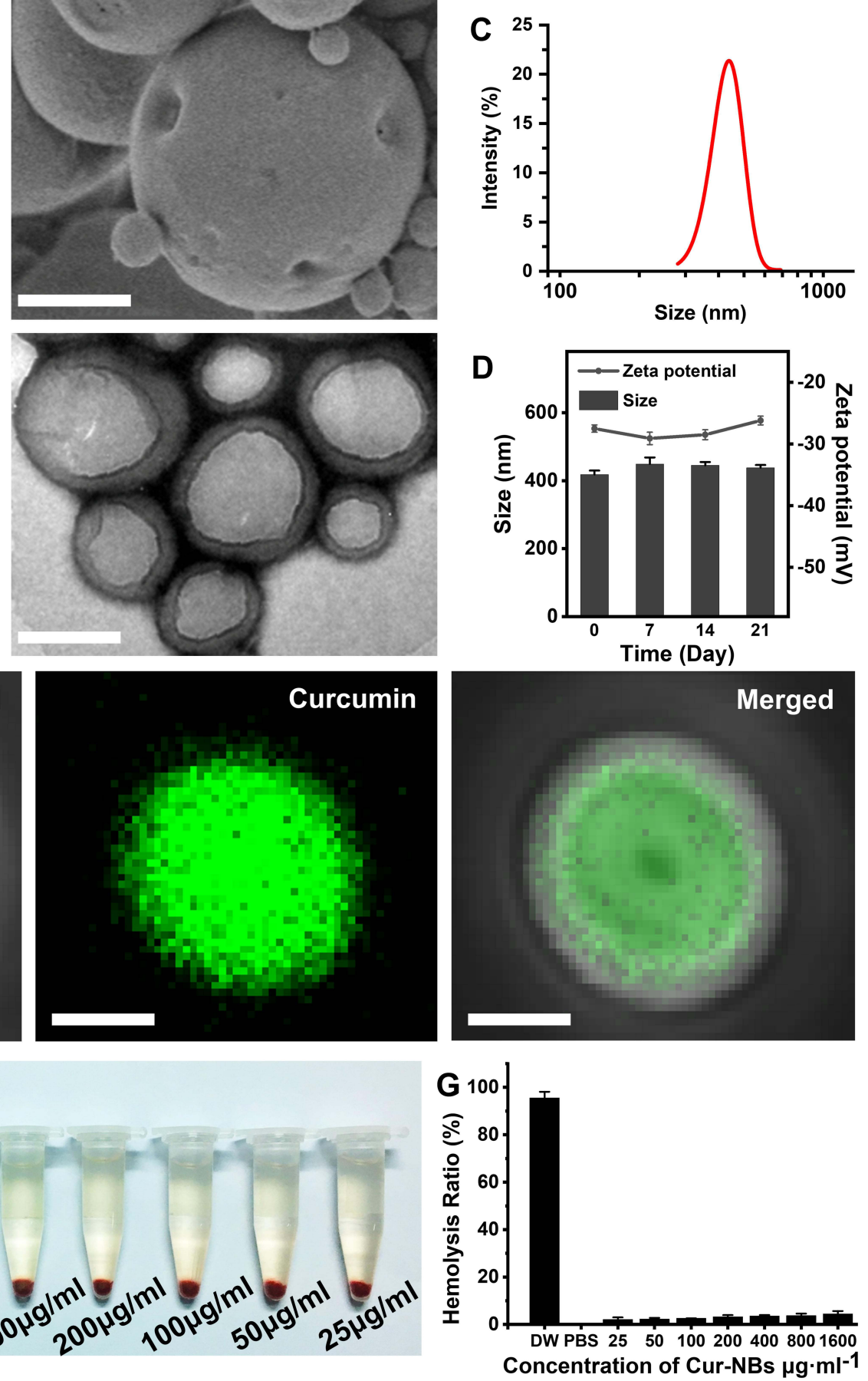

Figure 3 Characterization of Cur-NBs. (A) SEM of Cur-NBs after lyophilization. $500 \mathrm{~nm}$ (left scale bar), $250 \mathrm{~nm}$ (right scale bar). (B) TEM image of Cur-NBs. I00 nm (left scale bar), $200 \mathrm{~nm}$ (right scale bar). (C) DLS particle-size distributions of Cur-NBs. (D) The zeta potential and size changes of Cur-NBs measured within 3 weeks. (E) Observation of Cur-NBs under confocal fluorescence microscope (from left to right: bright field diagram, shell and internal cavity containing curcumin fluorescence, merged). Scale bar is $200 \mathrm{~nm}$. (F and G) Hemolysis rate of Cur-NBs at different concentrations (25, 50, 100, 200, 400, 800, 1600 $\mu \mathrm{g} / \mathrm{mL})$.

intravenously injected via tail vein and could last for more than 3 min (Figure 5D and E). By contrast, PBS injection via the same way cannot produce any contrast-enhanced signals. These results demonstrated Cur-NBs have a good ultrasound contrast imaging capability in vitro and in vivo.

\section{Ultrasound-Mediated BBB Opening with Cur-NBs}

To determine whether Cur-NBs combined with LIFU could open the blood brain-barrier, adult male wild-type mice (C57/BL6J) were used and exposed to different acoustic 

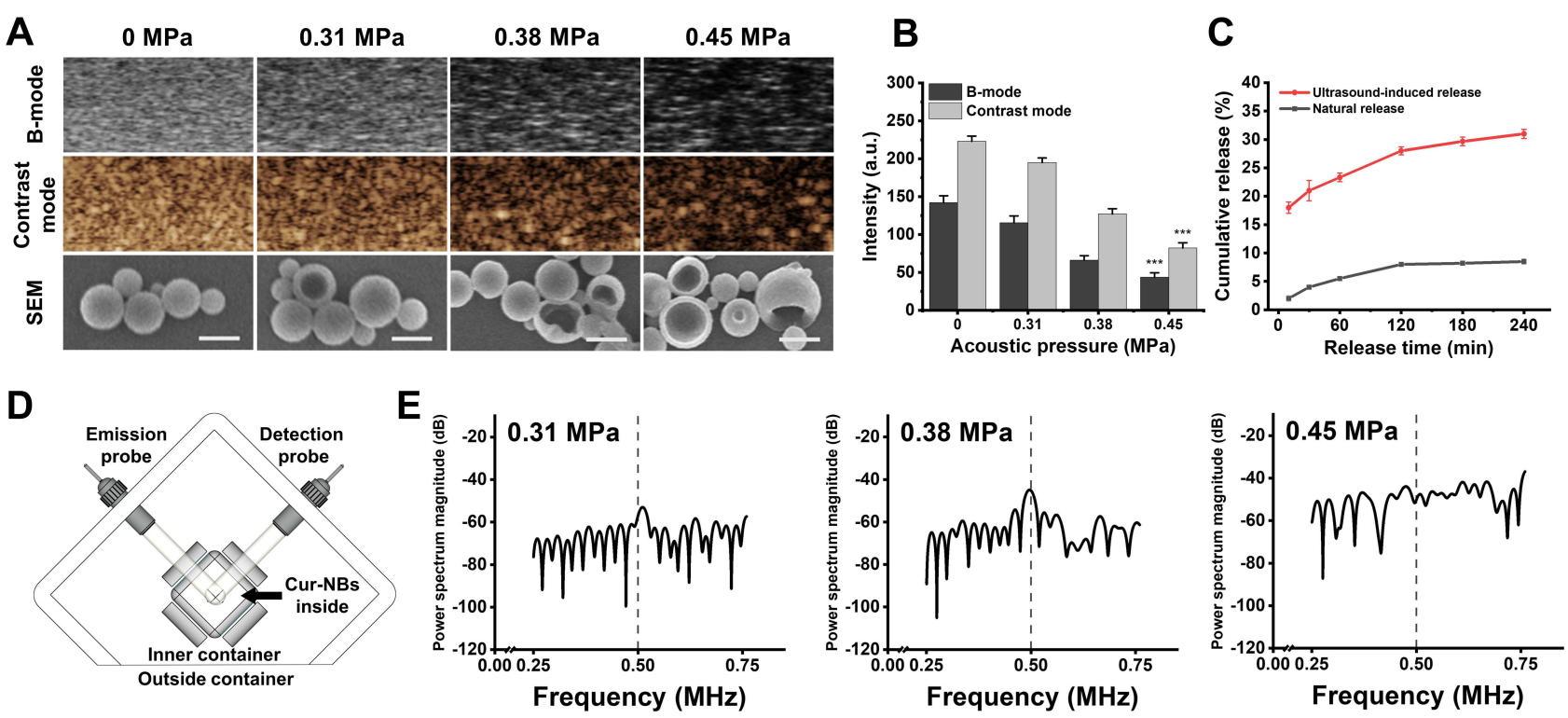

Figure 4 Ultrasound-mediated destruction of Cur-NBs and drug release. (A) Destruction of Cur-NBs at various acoustic intensities (0, $0.3 \mathrm{I}, 0.38,0.45 \mathrm{MPa})$ and their images evaluated in B-mode, contrast mode and SEM. Scale bar is $250 \mu \mathrm{m}$. (B) Quantitative analysis of b-mode and contrast mode signal intensities under various acoustic intensities. ***P $<0.001$ vs $0 \mathrm{MPa}$ group. (C) The time curve of curcumin release from Cur-NBs in the natural state or ultrasound destruction. Data were represented as mean \pm SD. (D) Schematic illustration of sub-harmonic detect setup. (E) The characteristics of sub-harmonic waves were detected at various acoustic intensities (0.3I, 0.38 , and $0.45 \mathrm{MPa}$ ).

pressures after intravenous injection of Cur-NBs and Evans blue (EB) via tail vein (Figure 6A). Figure 6B reveals that Cur-NBs combined with ultrasound could effectively open the BBB at various suitable acoustic pressures. Significant enhanced penetrations were observed after ultrasoundmediated BBB opening treatment when the acoustic pressures were more than 0.31 MPa (Figure 6C). Quantitatively, EB amount penetrated the brain after BBB opening at 0.45 $\mathrm{MPa}$ was 2.04-, 11.24-fold higher than at $0.38 \mathrm{MPa}$ and $0.31 \mathrm{MPa}$, respectively. No Evans blue could be delivered into the brain without ultrasound irradiation. Similarly, curcumin amount delivered into the brain at $0.45 \mathrm{MPa}$ acoustic pressure was also significantly more than at 0.38 $\mathrm{MPa}$ and $0.31 \mathrm{MPa}$ groups (Figure 6D). Interestingly, more curcumin were found to be delivered into the deep-seated brain region such as the cerebral cortex or corpus striatum at $0.45 \mathrm{MPa}$, with 1.37- and 1.45-fold higher than at 0.38 $\mathrm{MPa}$ and $0.31 \mathrm{MPa}$ groups. Thus, LIFU-induced BBB opening can deliver curcumin into the brain including deep-seated brain regions and would be used to enhance the efficacy of curcumin.

\section{Efficacy Evaluation of Cur-NBs Against PD}

Next, we further examined the treatment effect against PD. The sub-acute mouse PD model was built through daily intravenous administration with MPTP for 7 days. Curcumin was delivered once every two days through LIFU-mediated BBB opening with Cur-NBs. The behavioral tests were performed at day 7 and day 21 (Figure 7A). Our results showed that all mice groups with MPTP treatment showed poor neuromuscular coordination, showing that the retention time on the rotating rod was significantly reduced at day 7 and day 21 in comparison to wide-type control group (Figure 7B). However, the mice received with Cur-NBs + LIFU were able to stay on the rotarod significantly longer time than only Cur-NBs, only LIFU and PD model groups. Additionally, compared with retention time at 7 days, the mice treated with Cur$\mathrm{NBs}+$ LIFU stayed significantly longer on the rotarod at 21 days. But there were no significant differences in rotary rod test between 7 days and 21 days for only Cur-NBs, only LIFU and PD model groups. Also, we used the poleclimbing test to further evaluate the motor abilities of PD mouse. MPTP administration resulted in significant loss of agile movement and prolonged their total trial time compared with wide-type control group (Figure 7C). However, mice in Cur-NBs + LIFU group showed significant advantage of motor abilities, with trial time shorter than only Cur-NBs, only LIFU and PD model groups. Thus, our results indicated brain delivery of curcumin through LIFU-mediated BBB opening with Cur-NBs could relieve PD symptoms. 
A

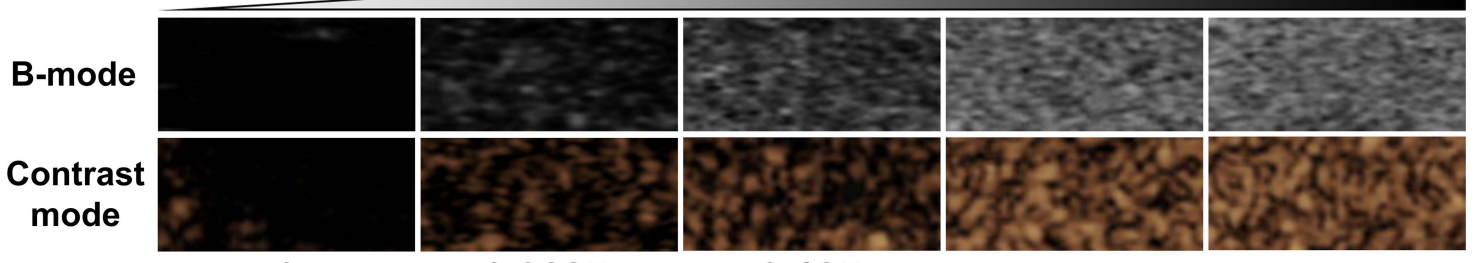

0

0.0625

0.125

0.25

0.5

B

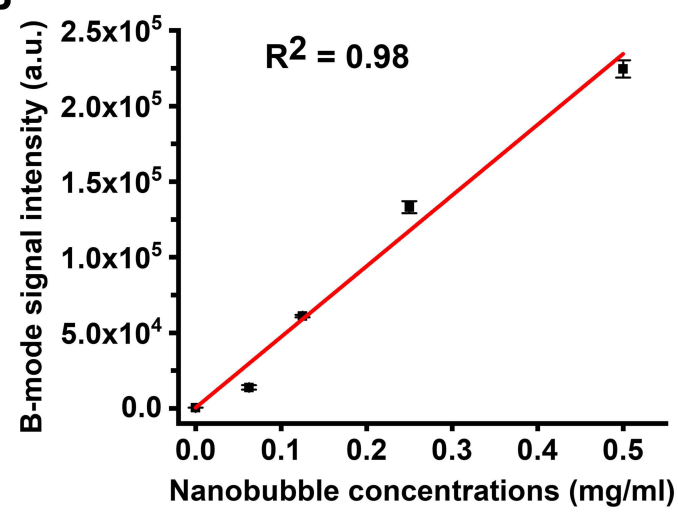

D

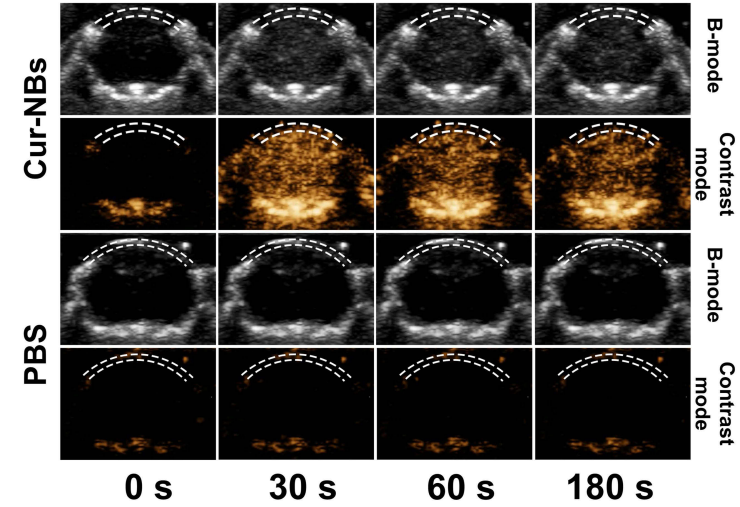

C

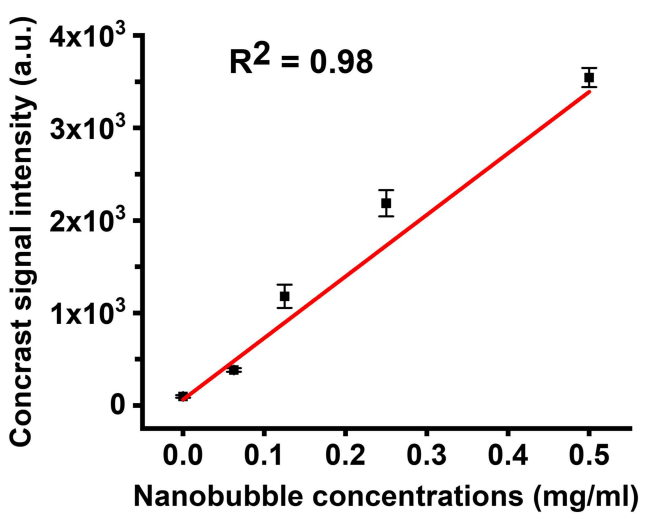

E

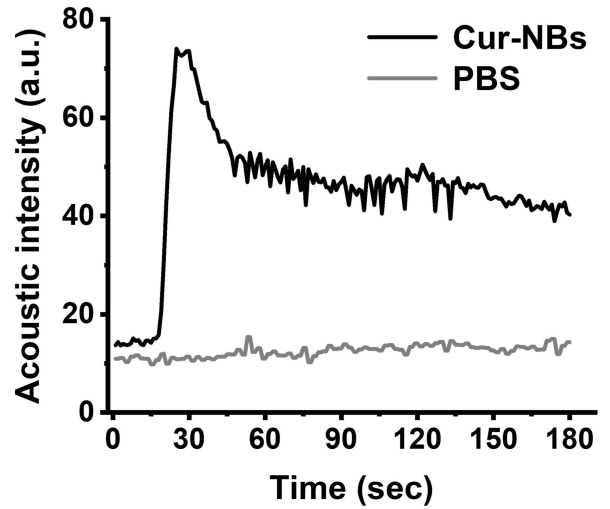

Figure 5 In vitro and in vivo acoustic imaging of Cur-NBs. (A) Ultrasound images of Cur-NBs at different concentrations $(0.0625 \mathrm{mg} / \mathrm{mL}, 0.125 \mathrm{mg} / \mathrm{mL}, 0.25 \mathrm{mg} / \mathrm{mL}, 0.5 \mathrm{mg} / \mathrm{mL})$ in B-mode and nonlinear contrast mode in vitro. (B) Quantification of the acoustic signals in B-mode $(n=3)$. (C) Quantification of the acoustic signals in nonlinear contrast mode ( $n$ = 3). (D) The acoustic signals of intracranial contrast imaging of injected Cur-NBs of PBS (the white dotted line is the removed skull). (E) The time curve of acoustic signals with injected Cur-NBs or PBS.

\section{Discussion}

In recent years, with the development of polymer materials and nanoscience, ultrasonic contrast agent with nanoscale trend to be popular due to their longer circulation time and higher surface area to carry drugs. ${ }^{40,41}$ In this study, we have fabricated the curcumin-loaded Lipid-PLGA nanobubbles (Cur-NBs) to deliver the curcumin into the brain for PD treatment by a modified double emulsion solvent evaporation method. Compared with conventional lipid microbubbles, our nanobubble possesses some obvious advantages. Firstly, Cur-NBs have a nanoscale particle size and relative uniform particle size distribution. This feature not only endows these bubbles with more stable drug formulation but also gives them more uniform responsiveness to US excitation. Secondly, the bubble shell was made of LipidPLGA polymers, having a harder shell structure than that of lipid bubbles, but softer shell than that of pure PLGA bubbles. ${ }^{42}$ That would make it possible to integrate the advantages of lipid bubbles and PLGA bubbles. As shown in Figures 4 and 5, our Cur-NBs not only had an excellent ultrasound contrast imaging capability but also possessed a good performance in US-triggered drug release property. 
A

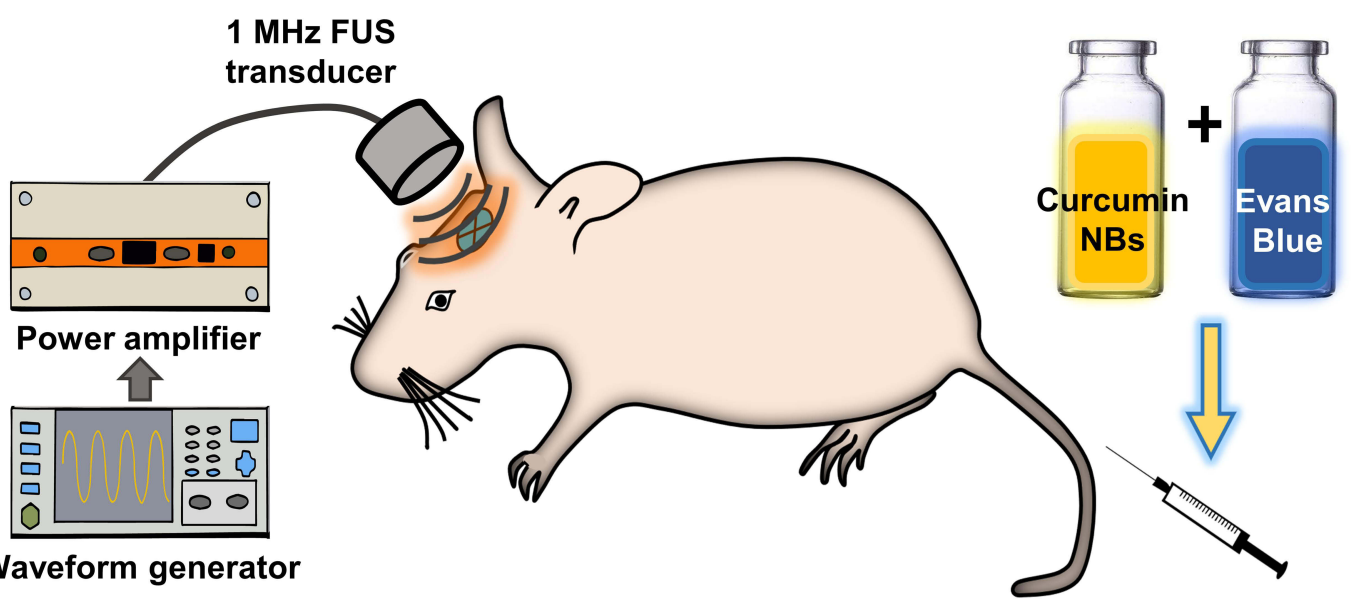

B

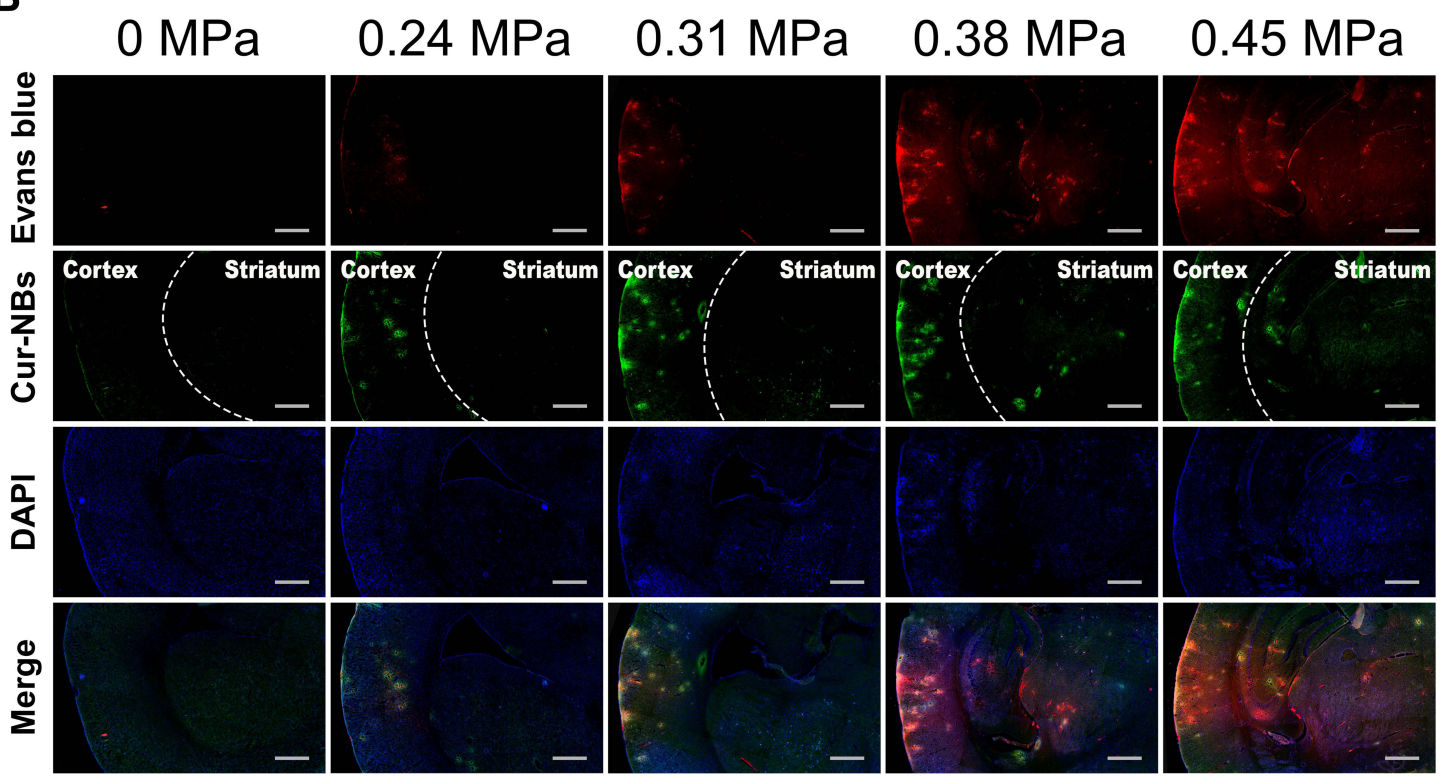

C

D
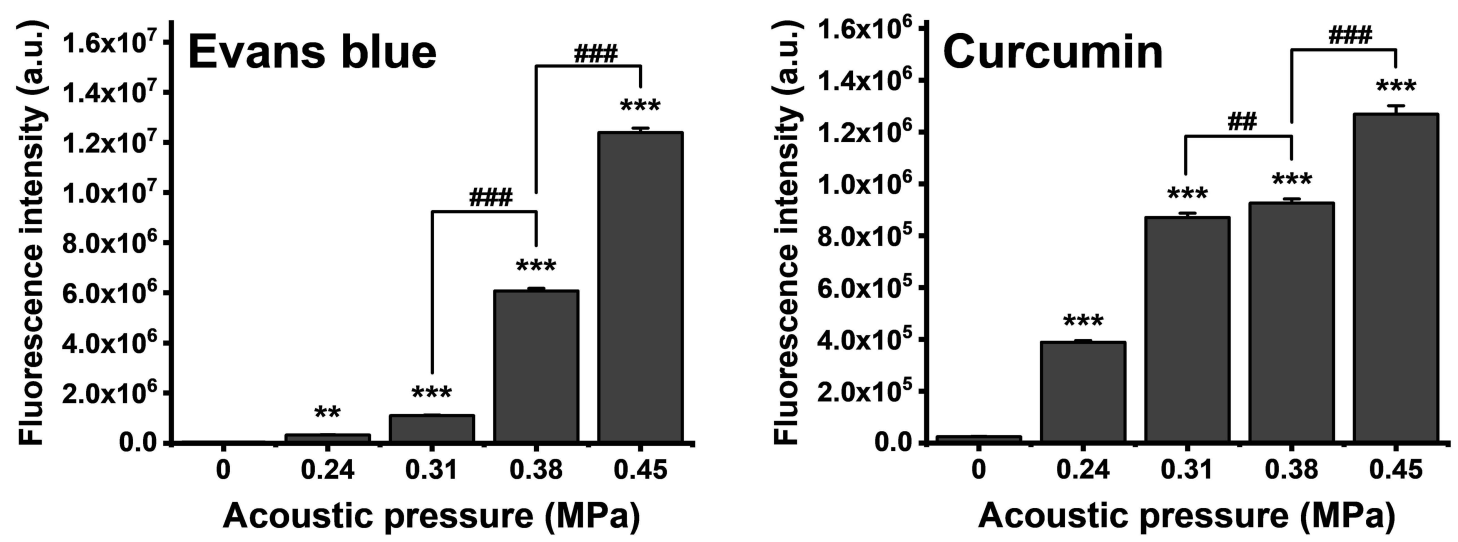

Figure 6 The BBB opening and drug permeability by Cur-NBs combined with LIFU. (A) Schematic representation of the BBB opening by Cur-NBs combined with LIFU. (B) Evaluation of BBB opening at the various acoustic intensities $(0,0.24,0.31,0.38$ and $0.45 \mathrm{MPa})$ by Cur-NBs combined with LIFU. (Cortex: cerebral cortex; Striatum: corpus striatum). Scale bar is $250 \mu \mathrm{m}$. (C and D) Quantitative analysis of fluorescent intensities of Evans blue and curcumin for each group. $* * \mathrm{P}<0.0 \mathrm{l}$ and $* * * \mathrm{P}<0.00 \mathrm{I}$ vs $0 \mathrm{MPa}$ group, ${ }^{\#} P<0.01$ and ${ }^{\# \# P} P<0.001$ between two groups. 
A

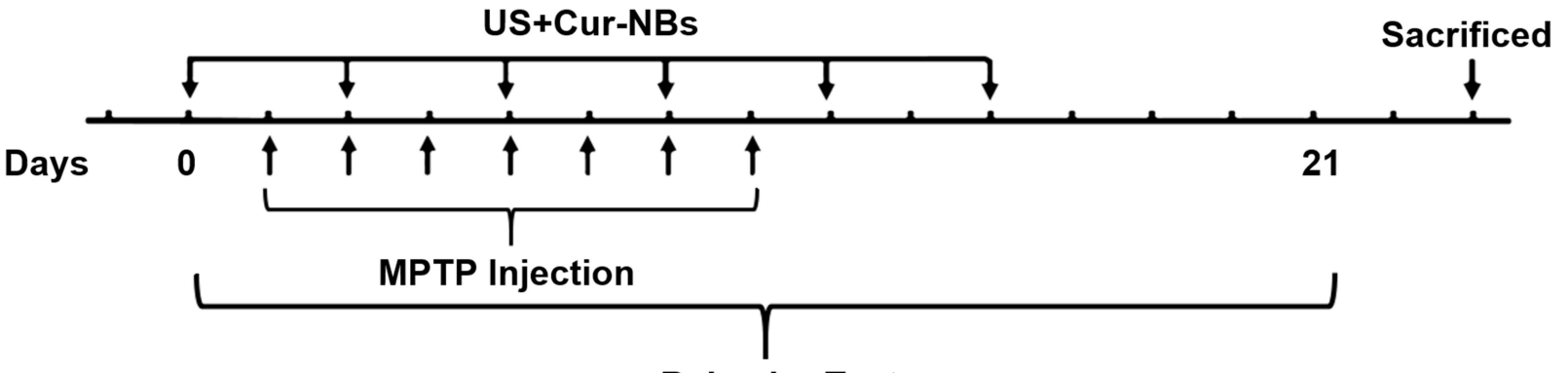

Behavior Test

B

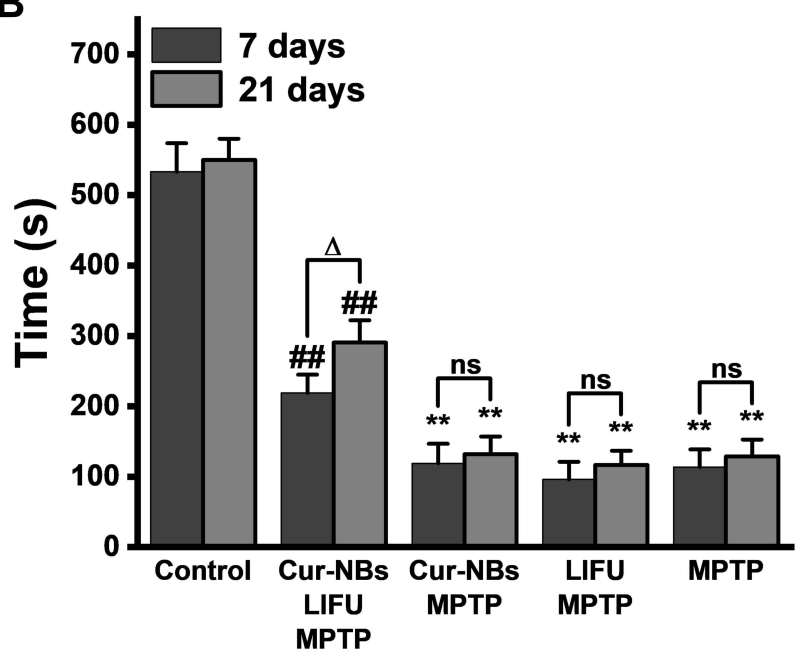

Rotary rod test
C

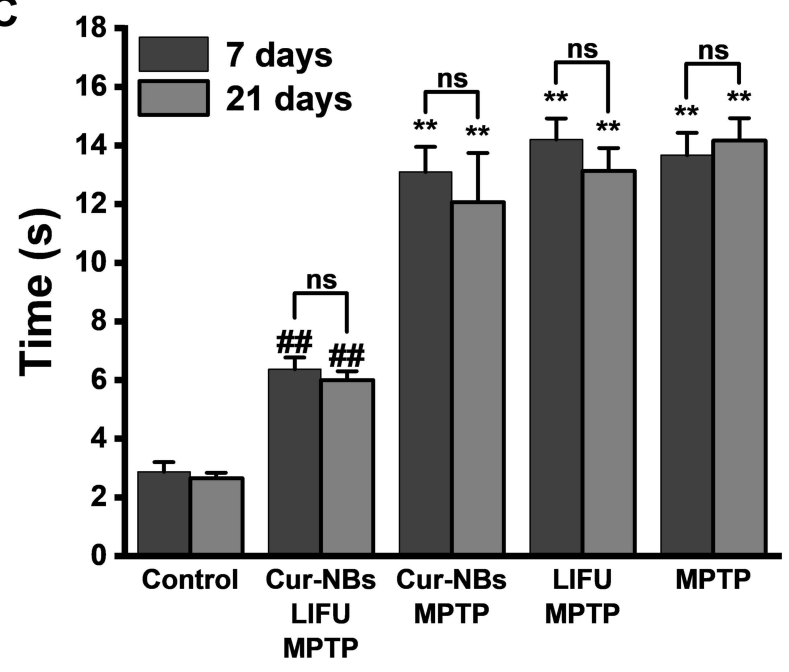

Pole-climbing test

Figure 7 The behavior analysis. (A) The treatment regimen schematic of MPTP-induced mouse model of Parkinson's disease ( $n=6$ per group). (B) Rotatory-rod test of PD and healthy control mice at 7 and 21 days after the first MPTP injection ( $n=6$ per group). (C) Pole-climbing test of PD and healthy control mice at 7 and 21 days after the first MPTP injection ( $n=6$ per group). ${ }^{* * P}<0.01$ vs control group, ${ }^{\#} P<0.01$ vs MPTP group, ${ }^{\Delta} P<0.05$ between two groups, ns $=$ not significant $(P>0.05)$ between two groups.

Meanwhile, the Cur-NBs showed better pressure resistance under the moderate-to-high acoustic energy, indicating that more stable cavitation rather than inertial cavitation would occur when they expose to US. It would be valuable for them in BBB opening because stable cavitation is safer than inertial cavitation. ${ }^{43}$ Less extreme physical reactions such as microjet and shock wave would produce, greatly alleviating the damage of bubble cavitation to brain. ${ }^{44,45}$ In addition, we prepared PEG-modified curcumin solid dispersion using melt-crystallization and encapsulated into the cavity of NBs, which may prevent them from drug leaking. Compared with the pure Curcumin and PM-SD, the aqueous solubility of curcumin dispersed on SD-Cur was largely enhanced improved by using polyethylene glycol 6000 as a hydrophilic carrier. It would improve the availability of drugs since the soluble curcumin is more easily released from the cavity of NBs than their lipid form.
Since the recognized mechanism of LIFU in bloodbrain barrier opening is predominantly cavitation effect, the physical events such as microjet and blasting shock wave associated with inertial cavitation may cause capillary damage or edema of fragile brain tissue, triggering a series of debates on the safety of LIFU on brain tissue. ${ }^{46}$ Steady-state cavitation means that the cavitation bubble only expands or shrinks under low acoustic pressure. This instantly expanded but unruptured bubble mechanically stretches the blood vessel wall in the local part of the brain tissue, which widens the tight connection of various microcirculation endothelial cells, increases the membrane pores of endothelial cells, and induces transcellular huff and puff. $^{47}$ To assay the most appropriate ultrasonic parameters, the acoustic energy parameters were optimized in this study. Our results found some fractured holes would generate on the bubble shell of Cur-NBs and bubble 
architecture did remain after LIFU irradiation. It is largely different cavitation from lipid bubbles, in which it is collapsed into lots of debris. So numerous microjet and shock wave would be avoidable for Cur-NBs at a high acoustic pressure when lipid bubbles produce inertial cavitation. ${ }^{48}$ In addition, the better solubilization of curcumin may also improve its bioavailability and decrease the risk of dangerous chemical agents.

In our study, our results demonstrated that Cur-NBs combined with LIFU could selectively open the blood brain-barrier and deliver the curcumin into the deepseated brain regions at the optimized acoustic parameters. It may be obviously beneficial to the delaying or reversing the disease progression for curcumin in our Parkinson mouse model. ${ }^{49}$ As the animal experiment shown above, mice in Cur-NBs + LIFU group performed significantly better efficacy in the rotating rod and pole-climbing tests of PD mice than only Cur-NBs, only LIFU and PD model groups, indicating brain delivery of curcumin through LIFU-mediated BBB opening could relieve PD symptoms.

\section{Conclusion}

Our study has successfully synthesized Cur-NBs which contained curcumin in the cavity of Lipid-PLGA nanobubbles. The resulting Cur-NBs performed excellent contrast imaging and can be used for LIFU-mediated BBB opening to locally deliver the curcumin into diseased brain regions. A significant improved efficacy was achieved in the Parkinson C57BL/6J mice model. Our study provides a platform for these potential drugs which are difficult to cross the $\mathrm{BBB}$ to treat $\mathrm{PD}$ disease or other CNS diseases.

\section{Abbreviations}

PD, Parkinson's disease; BBB, blood-brain barrier; CurNBs, curcumin-loaded Lipid-PLGA nanobubbles; LIFU, Low-intensity focused ultrasound; NBs, nanobubbles; CNS, central nervous system; SNC, substantia nigra pars compacta; a-syn, alpha-synuclein; MBs, microbubbles; PLGA, poly(lactide-co-glycolide); DSPC, 1,2-distearoyl-sn-glycero-3-phosphatidylcholine; DSPE-PEG2000, 1,2-distearoyl-sn-glycero-3-phosphoethanola-mine-N-[methoxy (polyethylene glycol)-2000]; (NH4)HCO3, ammonium bicarbonate; PEG-6000, polyethylene glycol 6000; SD, solid dispersion; PM, physical mixture; SD-Cur, PEG-modified curcumin solid dispersion; PM-Cur, curcumin-PEG6k physical mixture; DCM, dichloromethane; PVA, polyvinyl alcohol; SEM, scanning electron microscope; TEM, transmission electron microscope; DLS, dynamic light scattering; RBC, red blood cells; MPTP, 1-Methyl-4-phenyl1,2,3,6-tetrahydropyridine; PCD, passive cavitation detection; EB, Evans blue.

\section{Ethics Approval}

All animal work was performed according to Laboratory animal-Guideline for ethical review of animal welfare (the National Standard of the People's Republic of China, GB/T 35892-2018) and the ethics approval was obtained from the animal welfare committee of the Shenzhen Institutes of Advance Technology, the Chinese Academy of Sciences (approval no. SIAT-IRB-180208YGS-YF-A0442). Female C57BL6 mice were housed in a barrier-housing facility meeting national standards (Laboratory Animals: Requirements of Environment and Housing Facilities [GB 14925-2010]).

\section{Acknowledgments}

The authors gratefully acknowledge support for this research from the National Key R\&D Program of China (2018YFC0115900, 2020YFA0908800), National Natural Science Foundation of China (81871376, 81660381, 81371931,81974323 and 81301240), the Natural Science Foundation of Guangdong Province, China (201804010082), Guangdong Innovation Platform of Translational Research for Cerebrovascular Diseases, and Shenzhen Science and Technology Innovation Committee (JCYJ2019 0812171820731 and ZDSYS201802061806314), Shenzhen Strategic Emerging Industries Project (CXZZ20130 517095548798), Guangzhou Science and Technology Program Project (201804010082).

\section{Author Contributions}

Yiran Yan and Yan Chen contributed equally to this work and shared co-first authorship. Yiran Yan performed the experiment and wrote the manuscript. Liming Song, HaiFeng Liang, Zhiwen Su contributed to data interpretation and modified the experimental procedures. Zhongxun Liu and Wanting Niu conducted the synthesis of Cur-NBs and supporting techniques. Feiyan Cai measured the acoustic pressure distribution. Yan Chen, Bo Yu, and Fei Yan proposed the hypothesis, approved the data analysis, and revised the manuscript. All authors contributed to data analysis, drafting or revising the article, gave final approval of the version to be published, agreed to the 
submitted journal, and agreed to be accountable for all aspects of the work.

\section{Disclosure}

The authors report no conflicts of interest in this work.

\section{References}

1. Makin S. Pathology: the prion principle. Nature. 2016;538(7626): S13-S16. doi:10.1038/538S13a

2. Lee BE, Kim HY, Kim H, et al. O-GlcNAcylation regulates dopamine neuron function, survival and degeneration in Parkinson disease. Brain. 2020;143(12):3699-3716. doi:10.1093/brain/awaa320

3. Chung E, Choi Y, Park J, et al. Intracellular delivery of Parkin rescues neurons from accumulation of damaged mitochondria and pathological alpha-synuclein. Sci Adv. 2020;6(18):a1193. doi:10.1126/sciadv.aba1193

4. Pinto M, Fernandes C, Martins E, et al. Boosting drug discovery for Parkinson's: enhancement of the delivery of a monoamine oxidase-B inhibitor by brain-targeted PEGylated polycaprolactone-based nanoparticles. Pharmaceutics. 2019;11(7):331. doi:10.3390/pharmaceuti cs11070331

5. Kono Y, Colley T, To M, et al. Cigarette smoke-induced impairment of autophagy in macrophages increases galectin- 8 and inflammation. Sci Rep. 2021;11(1):335. doi:10.1038/s41598-020-79848-0

6. Liu L, Liao X, Wu H, Li Y, Zhu Y, Chen Q. Mitophagy and its contribution to metabolic and aging-associated disorders. Antioxid Redox Signal. 2020;32(12):906-927. doi:10.1089/ars.2019.8013

7. Alimohammadi E, Khedri M, Miri JA, Maleki R, Rezaian M. Graphene-based nanoparticles as potential treatment options for Parkinson's disease: a molecular dynamics study. Int J Nanomedicine. 2020;15:6887-6903. doi:10.2147/IJN.S265140

8. Han F, Baremberg D, Gao J, et al. Development of stem cell-based therapy for Parkinson's disease. Transl Neurodegener. 2015;4(1):16. doi:10.1186/s40035-015-0039-8

9. Li CW, Li LL, Chen S, Zhang JX, Lu WL. Antioxidant nanotherapies for the treatment of inflammatory diseases. Front Bioeng Biotechnol. 2020;8:200. doi:10.3389/fbioe.2020.00200

10. Robinson M, Lou J, Mehrazma B, Rauk A, Beazely M, Leonenko Z. Pseudopeptide amyloid aggregation inhibitors: in silico, single molecule and cell viability studies. Int $J$ Mol Sci. 2021;22(3):1051. doi:10.3390/ijms22031051

11. Bodratti AM, Alexandridis P. Amphiphilic block copolymers in drug delivery: advances in formulation structure and performance. Expert Opin Drug Deliv. 2018;15(11):1085-1104. doi:10.1080/ 17425247.2018.1529756

12. Ferraris C, Cavalli R, Panciani PP, Battaglia L. Overcoming the blood-brain barrier: successes and challenges in developing nanoparticle-mediated drug delivery systems for the treatment of brain tumours. Int J Nanomed. 2020;15:2999-3022. doi:10.2147/ IJN.S231479

13. Kinfe T, Stadlbauer A, Winder K, Hurlemann R, Buchfelder M. Incisionless MR-guided focused ultrasound: technical considerations and current therapeutic approaches in psychiatric disorders. Expert Rev Neurother. 2020;20(7):687-696. doi:10.1080/14737175.20 20.1779590

14. McDannold N, Zhang Y, Supko JG, et al. Acoustic feedback enables safe and reliable carboplatin delivery across the blood-brain barrier with a clinical focused ultrasound system and improves survival in a rat glioma model. Theranostics. 2019;9(21):6284-6299. doi: $10.7150 /$ thno. 35892
15. Meng L, Liu X, Wang Y, et al. Sonoporation of cells by a parallel stable cavitation microbubble array. Adv Sci. 2019;6(17):1900557. doi:10.1002/advs.201900557

16. Lin $\mathrm{Y}$, Chen $\mathrm{H}$, Chen $\mathrm{H}$, et al. Ultrastructural changes associated with the enhanced permeability of the round window membrane mediated by ultrasound microbubbles. Front Pharmacol. 2020;10:1580. doi:10.3389/fphar.2019.01580

17. Do HD, Couillaud BM, Doan BT, Corvis Y, Mignet N. Advances on non-invasive physically triggered nucleic acid delivery from nanocarriers. Adv Drug Deliv Rev. 2019;138:3-17. doi:10.1016/j.addr.2018.10.006

18. Park T, Mustafaoglu N, Herland A, et al. Hypoxia-enhanced blood-brain barrier chip recapitulates human barrier function and shuttling of drugs and antibodies. Nat Commun. 2019;10(1):2621. doi:10.1038/s41467-019-10588-0

19. Shen Z, Liu T, Li Y, et al. Fenton-reaction-acceleratable magnetic nanoparticles for ferroptosis therapy of orthotopic brain tumors. ACS Nano. 2018;12(11):11355-11365. doi:10.1021/acsnano.8b06201

20. Sukumar UK, Bose R, Malhotra M, et al. Intranasal delivery of targeted polyfunctional gold-iron oxide nanoparticles loaded with therapeutic microRNAs for combined theranostic multimodality imaging and presensitization of glioblastoma to temozolomide. Biomaterials. 2019;218:119342. doi:10.1016/j.biomaterials.2019.119342

21. Song K, Harvey BK, Borden MA. State-of-the-art of microbubble-assisted blood-brain barrier disruption. Theranostics. 2018;8(16):4393-4408. doi: $10.7150 /$ thno. 26869

22. Pysz MA, Willmann JK. Targeted contrast-enhanced ultrasound: an emerging technology in abdominal and pelvic imaging. Gastroenterology. 2011;140(3):785-790. doi:10.1053/j.gastro.2011.01.027

23. Ehlerding EB, Grodzinski P, Cai W, Liu CH. Big potential from small agents: nanoparticles for imaging-based companion diagnostics. ACS Nano. 2018;12(3):2106-2121. doi:10.1021/acsnano.7b07252

24. Lammertink BH, Bos C, Deckers R, Storm G, Moonen CT, Escoffre JM. Sonochemotherapy: from bench to bedside. Front Pharmacol. 2015;6:138. doi:10.3389/fphar.2015.00138

25. Zhai K, Brockmüller A, Kubatka P, Shakibaei M, Büsselberg D. Curcumin's beneficial effects on neuroblastoma: mechanisms, challenges, and potential solutions. Biomolecules. 2020;10(11):1469. doi:10.3390/biom 10111469

26. Nguyen C, Pandey S. Exploiting mitochondrial vulnerabilities to trigger apoptosis selectively in cancer cells. Cancers. 2019;11 (7):916. doi:10.3390/cancers11070916

27. Naksuriya O, Okonogi S, Schiffelers RM, Hennink WE. Curcumin nanoformulations: a review of pharmaceutical properties and preclinical studies and clinical data related to cancer treatment. Biomaterials. 2014;35(10):3365-3383. doi:10.1016/j. biomaterials.2013.12.090

28. Li H, Sureda A, Devkota HP, et al. Curcumin, the golden spice in treating cardiovascular diseases. Biotechnol Adv. 2020;38:107343. doi:10.1016/j.biotechadv.2019.01.010

29. Yavarpour-Bali H, Ghasemi-Kasman M, Pirzadeh M. Curcuminloaded nanoparticles: a novel therapeutic strategy in treatment of central nervous system disorders. Int $J$ Nanomed. 2019;14:4449-4460. doi:10.2147/IJN.S208332

30. Pandey N, Strider J, Nolan WC, Yan SX, Galvin JE. Curcumin inhibits aggregation of alpha-synuclein. Acta Neuropathol. 2008;115 (4):479-489. doi:10.1007/s00401-007-0332-4

31. Wang Z, Yang C, Liu J, et al. A curcumin derivative activates TFEB and protects against Parkinsonian neurotoxicity in vitro. Int $J \mathrm{Mol}$ Sci. 2020;21(4):1515. doi:10.3390/ijms21041515

32. Huerta-ángeles G, Brandejsová M, Novotný J, et al. Grafting of steroids to hyaluronan towards the design of delivery systems for antioxidants: the role of hydrophobic core. Carbohydr Polym. 2018;193:383-392. doi:10.1016/j.carbpol.2018.04.021 
33. Leksiri S, Wasana D, Wadu P, Vajragupta O, Rojsitthisak P, Towiwat P. Co-administration of pregabalin and curcumin synergistically decreases pain-like behaviors in acute nociceptive pain murine models. Molecules. 2020;25(18):4172. doi:10.3390/molecules 25184172

34. Davis MT, Potter CB, Walker GM. Downstream processing of a ternary amorphous solid dispersion: the impacts of spray drying and hot melt extrusion on powder flow, compression and dissolution. Int J Pharm. 2018;544(1):242-253. doi:10.1016/j.ijpharm.2018.04.038

35. Chaturvedi K, Shah HS, Nahar K, Dave R, Morris KR. Contribution of crystal lattice energy on the dissolution behavior of eutectic solid dispersions. ACS Omega. 2020;5(17):9690-9701. doi:10.1021/ acsomega.9b03886

36. Sarabu S, Kallakunta VR, Bandari S, et al. Hypromellose acetate succinate based amorphous solid dispersions via hot melt extrusion: effect of drug physicochemical properties. Carbohydr Polym. 2020;233:115828. doi:10.1016/j.carbpol.2020.115828

37. King DA, Malloy MJ, Roberts AC, Haak A, Yoder CC, O'Brien WDJ. Determination of postexcitation thresholds for single ultrasound contrast agent microbubbles using double passive cavitation detection. J Acoust Soc Am. 2010;127(6):3449-3455. doi:10.1121/1.3373405

38. Sorasitthiyanukarn FN, Ratnatilaka NBP, Muangnoi C, Rojsitthisak P, Rojsitthisak P. Chitosan/alginate nanoparticles as a promising carrier of novel curcumin diethyl diglutarate. Int $J$ Biol Macromol. 2019;131:1125-1136. doi:10.1016/j.ijbiomac.2019.03.120

39. Snipstad S, Sulheim E, de Lange DC, et al. Sonopermeation to improve drug delivery to tumors: from fundamental understanding to clinical translation. Expert Opin Drug Deliv. 2018;15 (12):1249-1261. doi:10.1080/17425247.2018.1547279

40. Hysi E, Fadhel MN, Wang Y, et al. Photoacoustic imaging biomarkers for monitoring biophysical changes during nanobubble-mediated radiation treatment. Photoacoustics. 2020;20:100201. doi:10.1016/j. pacs.2020.100201

41. Bosch-Rué E, Diez-Tercero L, Giordano-Kelhoffer B, et al. Biological roles and delivery strategies for ions to promote osteogenic induction. Front Cell Dev Biol. 2021;8:614545. doi:10.3389/ fcell.2020.614545
42. Chen Y, Liang Y, Jiang P, Li F, Yu B, Yan F. Lipid/PLGA hybrid microbubbles as a versatile platform for noninvasive image-guided targeted drug delivery. ACS Appl Mater Interfaces. 2019;11 (45):41842-41852. doi:10.1021/acsami.9b10188

43. Dauba A, Delalande A, Kamimura HAS, et al. Recent advances on ultrasound contrast agents for blood-brain barrier opening with focused ultrasound. Pharmaceutics. 2020;12(11):1125. doi:10.3390/ pharmaceutics 12111125

44. Li M, Li J, Zhu C. Effect of ultrasound pretreatment on enzymolysis and physicochemical properties of corn starch. Int J Biol Macromol. 2018;111:848-856. doi:10.1016/j.ijbiomac.2017.12.156

45. Li R, Lin Z, Zhang Q, et al. Injectable and in situ-formable thiolated chitosan-coated liposomal hydrogels as curcumin carriers for prevention of in vivo breast cancer recurrence. ACS Appl Mater Interfaces. 2020;12(15):17936-17948. doi:10.1021/acsami.9b21528

46. Magnin R, Rabusseau F, Salabartan F, et al. Magnetic resonance-guided motorized transcranial ultrasound system for blood-brain barrier permeabilization along arbitrary trajectories in rodents. J Ther Ultrasound. 2015;3(1):22. doi:10.1186/s40349-0150044-5

47. Hu S, Zhang X, Unger M, Patties I, Melzer A, Landgraf L. Focused ultrasound-induced cavitation sensitizes cancer cells to radiation therapy and hyperthermia. Cells Basel. 2020;9(12):2595. doi:10.3390/cells9122595

48. Lee JH, Moon H, Han H, et al. Antitumor effects of intra-arterial delivery of albumin-doxorubicin nanoparticle conjugated microbubbles combined with ultrasound-targeted microbubble activation on VX2 rabbit liver tumors. Cancers. 2019;11(4):581. doi:10.3390/ cancers 11040581

49. Song KH, Fan AC, Hinkle JJ, Newman J, Borden MA, Harvey BK. Microbubble gas volume: a unifying dose parameter in blood-brain barrier opening by focused ultrasound. Theranostics. 2017;7 (1):144-152. doi:10.7150/thno. 15987
International Journal of Nanomedicine

\section{Publish your work in this journal}

The International Journal of Nanomedicine is an international, peerreviewed journal focusing on the application of nanotechnology in diagnostics, therapeutics, and drug delivery systems throughout the biomedical field. This journal is indexed on PubMed Central, MedLine, CAS, SciSearch ${ }^{\circledR}$, Current Contents ${ }^{\circledR} /$ Clinical Medicine,
Journal Citation Reports/Science Edition, EMBase, Scopus and the Elsevier Bibliographic databases. The manuscript management system is completely online and includes a very quick and fair peer-review system, which is all easy to use. Visit http://www.dovepress.com/ testimonials.php to read real quotes from published authors. 